\title{
Moody's Versus Siegel's Interpretation of the Near-Death Experience: An Evaluation Based on Recent Research
}

\author{
John C. Gibbs \\ Department of Psychology \\ Ohio State University
}

\begin{abstract}
Recent research provides a basis for evaluating Raymond Moody's (1975) versus Ronald Siegel's (1980) interpretations of the near-death experience (NDE). Whereas Moody had concluded that the NDE is ontologically valid, Siegel interpreted the NDE as a purely subjective hallucinatory phenomenon. The findings of two recent investigations (Ring, 1980; Sabom, 1982), both utilizing large samples and appropriate methodological and statistical controls, generally appear to be more consistent with Moody's than with Siegel's interpretation. Basic features of the NDE have been found not attributable to particular background or circumstantial influences; the NDE's likelihood and extensiveness are associated with degree of physical near-death; visual details of NDE recollections are substantiated by medical records and are more accurate than "recollections" roleplayed by non-NDE survivors; and the NDE is experienced as uniquely impactful and real.
\end{abstract}

In his widely noted 1980 essay, Ronald Siegel disputed Raymond Moody's (1975, 1977) interpretation of the near-death experience (NDE). Moody's interpretation was that the NDE may be ontologically valid, on the basis of which he suggested "that death is a separation of the mind from the body, and that mind does pass into other realms of existence at this point' (1975, p. 151). In other words, Moody inferred a veridicality to the NDE compatible with traditional notions of a soul and afterlife (although Moody specifically disclaimed that his work could "prove that there is life after death," (p. 5). In contrast, Siegel asserted that the phenomenon was not "objective" but rather "subjective,"' and specifically, the product of "dissociative hallucinatory activity of the brain" (p. 911), "triggered by a variety of stimuli that result in massive cortical disinhibition and autonomic arousal"' (p. 924).

The purpose of this article is to evaluate Moody's versus Siegel's interpretations in the light of two major research studies (as well as a survey) of the NDE. Contemporaneous with and subsequent to Siegel's 1980 essay, two investigators published their extensive 
research findings (cf. Gibbs, 1981). Moody had written: "I am fully aware that what I have done does not constitute a scientific study" (p. 181); both Kenneth Ring (1980) and Michael Sabom (1982) independently determined in the late 1970s after reading the Moody book to conduct follow-up research that would meet scientific standards. The general initial attitude of both Ring and Sabom was one of interest but also of frustration at the haphazard data collection and reporting in Life After Life; specifically, Ring was sympathetic (1980, p. 17), while Sabom was thoroughgoingly skeptical (pp. 3, 7.) Both sought to establish an empirical basis for closure with respect to certain issues raised or suggested by the Moody work. Further pertinent findings were provided by George Gallup Jr.'s recent (1982) survey research on the NDE.

Preliminary questions pertained to how common and consistently uniform the NDE actually is: Moody had surmised that the phenomenon is fairly common and consistent, and Siegel did not challenge these assumptions despite the fact that further research investigation was clearly needed. Since Moody's cases had accumulated largely through publicity from his ongoing work, he had no basis for providing even an approximate answer to the elementary question of how common such experiences are among near-death survivors. Accordingly, both Ring and Sabom avoided designation of the NDE as a criterion for data collection at the outset. The Ring team sought subjects strictly on the basis of having come close to death from "a serious illness, accident, or suicide attempt" (p. 27). The Sabom team contacted hospital patients "who had suffered a near-death crisis event" (p. 7), and in initially approaching the patient "would act as if only routine medical details were being sought" (p. 9). The Sabom team also collected additional cases referred by medical colleagues who had learned of their NDE interest, but these cases were analyzed separately from the main sample. The subjects in both studies included comparable numbers of males and females, and were mostly white adults (some blacks, some adolescents) of diverse occupational and religious backgrounds.

The general result: Somewhere between one-third and one-half of near-death survivors reported having had NDEs (49 out of 102 subjects in Ring's study, 34 out of 78 prospective cases in Sabom's study, 35 percent of Gallup's "near death survivor" respondents for a projected estimate of 8 million Americans). Although the NDE apparently is not universal, it seems to be common enough to support Moody's implicit claim that it is a widespread occurrence.

Another Moody claim simply accepted by Siegel was that the NDE is a reasonably coherent phenomenon, that its features are sufficiently 
consistent across many cases to comprise a unitary construct. Ring used Moody's (1975) 15 elements "as a provisional basis for grasping the core experience"' (p. 24), but concluded after studying his data that the NDE could be "meaningfully ordered" in terms of "five distinct stages" which, when "considered in sequence, form a coherent pattern ... the experience of (apparent) death in its developmental form" (p. 39). The five stages were: (1) a sense of peace and well being; (2) detachment from one's physical body; (3) entering a dark region (sometimes described as a tunnel); (4) seeing a brilliant light; and (5) entering the light or another realm of existence. The "stages" were reported in decreasing frequency, ranging from 60 percent of the total sample for stage 1 to 10 percent for stage 5. Ring found all of Moody's elements, although several (e.g., hearing a noise departing the body) were quite infrequent. Overall, Ring concluded that the NDE does constitute a unitary phenomenon.

Sabom reached the same conclusion, describing the NDE as "an ineffable sense of timeless reality occurring apart from one's physical body and associated with a pleasant understanding of death"' (p. 23). Sabom systematized Moody's 15 elements into ten, which he described as follows: (1) subjective sense of being dead; (2) feelings of calm or peace (but sometimes preceded by momentary feelings of sadness, loneliness, or fright); (3) sense of bodily separation; (4) observation and hearing of physical events; (5) passage into a dark region or void (sometimes described as a tunnel); (6) experiencing a replay of significant past life events; (7) perception of a brilliant source of light; (8) entering a region of scenic beauty; (9) encountering personages such as deceased relatives or spiritual figures; and (10) return to one's physical body. These elements were commonly found with the exception of element 6 , the life review, which was recollected by only a few respondents.

Instead of organizing the elements into five "stages," Sabom organized them into two "components": "autoscopic" (or selfvisualizing, usually from above the physical body ${ }^{2}$ ), and "transcendental" (a "deeper realm of the NDE," entailing "descriptions of objects and events that 'transcend' or surpass all earthly limits"; pp. 37, 41). Whereas elements 1 through 3 and 10 were common to both components, element 4 defined the autoscopic component and elements 5 through 9 (at least 1 of them) defined the transcendental component. One-third of Sabom's NDE cases entailed only the autoscopic component, 48 percent entailed only transcendental elements, and 19 percent elements of both, such that "the transcendental portion of the experience followed the autoscopic 
portion in a continuous, unbroken sequence"' (p. 52).

Although Siegel accepted-correctly, it turned out-Moody's suggestions that the NDE is reasonably common and coherent, Siegel explicitly or implicitly doubted the tenability of at least four other Moody suggestions consistent with Moody's conclusion that the NDE represents an authentic spiritual phenomenon: (1)that the NDE is not specific to any particular personal background or to circumstance of near-death; (2) that the NDE varies in depth or extensiveness as a function of seriousness of clinical death; (3) that the specific recollections reported actually took place during the near-death period; and (4) that NDE and aftereffects are distinct, especially that NDErs who have also experienced hallucinations specify the NDE only as "real." Consistent with Siegel's subjectivist interpretation of the NDE were his inferences that it entails idiosyncratic content and circumstantial influences, constitutes subjective memories and images, and has no unique effect on worldview or lifestyle. Each of these issues constitutes researchable questions, to which various findings by Ring and Sabom are pertinent.

\section{RELIABILITY OF THE NDE ACROSS DIVERSE BACKGROUND AND CIRCUMSTANTIAL VARIABLES}

Moody expressed "amazement" at "the great similarity in the (NDE) reports, despite the fact that they come from people of highly varied religious, social, and educational backgrounds" (1975, p. $15)$ as well as the fact that "the circumstances surrounding" the incidents "varied widely" (p.17). Indeed, "in quite a few instances, reports have come from persons who had no religious beliefs or training at all prior to their experiences, and their descriptions do not seem to differ in content from people who had quite strong religious beliefs" (1975, p. 141). On the other hand, Moody acknowledged that religious background and belief did shape the NDE " to some extent," e.g., "though the description of the being of light is invariable, the identity ascribed to it varies, apparently as a function of the religious background of the individual" (p. 140).

Siegel-although he accepted the coherence of the NDE phenomenon-asserted a much greater extent of background and circumstantial influence on NDE content:

The specific content of complex hallucinatory imagery is determined largely by set (expectations and attitudes) and setting (physical and psychological 
environment). For many dying and near-death experiences, sets (fear of approaching death, changes in body and mental functioning, etc.) and settings (hospital wards, accident scenes, etc.) can influence specific eschatological thoughts and images. (p. 926).

The research by Ring and Sabom suggests that, in general terms, Moody was closer to the mark on this issue. After conducting numerous statistical analyses, Ring concluded that the NDE "appears to be a remarkably robust phenomenon, cutting across a variety of situational, individual, and demographic factors"' (1980, p. 137; cf. Gallup, 1982, pp. 31-56). NDEs were reported with comparable frequency and depth irrespective of mode of near-death onset (illness, accident, to some extent suicide attempt ${ }^{3}$ ), belief background (religious denomination, degree of religiousness), and other person variables (age, sex, race, social class, and marital status). Similarly, Sabom found no frequency differences "between groups broken down according to age, area of residence, size of home community, religious background or frequency of church attendance. Moreover, groups at the highest and lowest levels of educational attainment reported these elements with similar frequency" (p. 59). Sabom also found no significant differences by location of near-death episode (inside or outside a hospital) and type of crisis event (cardiac arrest, coma, or accident) (p. 202). Finally, Sabom pointed out that "a major portion of the interviewing was done at a time when Life After Life and related media were not general knowledge in the rural populations of northern Florida (where most of the subjects reside)" (p. 158). Sabom concluded "that the content of the NDE is quite consistent among near-death survivors from the American culture ${ }^{4}$ with differing backgrounds"' (p. 60).

Although the NDE in general terms does not seem to be provincial to particular persons or circumstances-and indeed, as noted earlier, seems to be reliably characterizable in terms of the basic "stages" or "components" described respectively by Ring and Sabom-the idiosyncracies of specific content may be greater than Moody acknowledged, and closer to Siegel's emphasis. Siegel wrote: "Like hallucinations, visions of the afterlife are suspiciously similar to this world" (p. 924). An anomalous case noted by Sabom in a data appendix is consistent with Siegel's suspicions of the familiar and the idiosyncratic. Sabom's case $\# 21$, a white, blue-collar, protestant male aged 58, reported a transcendental-type NDE in which he apparently experienced a light (element 7 ) yet did not enter any region of scenic beauty (element 8 ) but instead reported an idiosyncratic version of element 9, encountering others: he sensed the "presence 
of four unknown nurses" and encountered "verbal interrogation by nurses about possible "subversive activities", (p. 210). Nor does such an experience sound particularly pleasant (element 2). ${ }^{5}$ (Admittedly, the respondent might have suspected this part of his NDE to be hallucinatory; see "Status and Effects Uniquely Attributable to NDEs" section, below.) Although case 21 was apparently quite anomalous, even one NDE case entailing such major idiosyncracies in specific content suggests that Siegel's assertion of the role of subjective expectancy (in the above case, paranoid anxieties?) cannot be altogether dismissed.

\section{ASSOCIATION OF THE NDE WITH CLINICAL DEATH}

Although Siegel did not explicitly challenge Moody's assumption that the NDE is associated with clinical death, his corticaldisinhibition explanation would not seem to be necessarily consistent with an assumption of neurophysiological depression or reduction during the experience. Indeed, Siegel considered NDEs, like any hallucination, to be "directly related to states of excitation and arousal of the central nervous system" (p. 925). Yet clinical death (unconsciousness as well as absence of reflexes, respiration, and pulse) could hardly be described as a state of "excitation and arousal of the central nervous system." If Siegel's hypothesis is correct, then one would doubt that the NDE could have occurred during actual clinical death, and certainly would anticipate a negative relationship between extensiveness or "depth" of the NDE and seriousness of the associated near-death crisis. Yet Ring found a significant positive correlation between ratings of the extensiveness of the NDE (1980, pp. 32-33) and ratings of closeness to physical death (1980, pp. 109-112). Similarly, Sabom found that NDEs were significantly more likely where the survivor's duration of unconsciousness (estimated from medical records) had been greater than 30 minutes, or where the method of resuscitation was intensive, that is, included "all measures (i.e., intravenous glucose, electrolytes, antibodies, etc.) used in critical nonacute comatose situations" (p. 58).

A weaker form of a hallucination hypothesis could point out that none of either Ring's or Sabom's cases entailed documented brain death, and hence the NDE could still be dependent upon some minimal electro-chemical activity of the brain. Indeed, Mark Woodhouse (1983) specifically recommended inquiry into whether NDEs can occur during "flat EEG's" as a critical approach to providing an empirically based evaluation of whether the NDE is an imaginal or veridical experience. Moody (1975, pp. 148-149) 
disputed the value of this kind of evidence, however, pointing out the problematic character of EEG assessment. Of course, even assuming that NDEs were found to be dependent upon minimal brain activity, one would be left with having to explain why NDEs were elaborated in proportion to reduced, rather than enhanced, neurophysiological arousal.

Siegel could retain his original hypothesis by assuming that the NDEs did in fact occur during states of excitation and arousal-but before or after the near-death crisis-and then incorrectly attributed to the near-death period. One approach to assessing this question is especially intriguing. Moody noted that many of his cases reported relatively unique visual details specific to the near-death period while out of body, and asserted that these details were in some instances independently corroborated by doctors and other witnesses. If this were true, and if these details were of a kind unlikely to be told to the survivor subsequently by witnesses, then one would have fairly strong evidence that the NDEs did in fact occur during the period of clinical death. Indeed, one would then be constrained to conclude that the autoscopic activity "really" occurred.

\section{VERIFIABILITY OF NDE AUTOSCOPIC RECOLLECTIONS}

Siegel's imaginal interpretation of NDE autoscopic remembrances entailed noting "the constructive aspect of hallucinations" and suggesting "a simple exercise":

Recall the last time you went swimming in the ocean. Now ask yourself if this memory includes a picture of yourself running along the beach or moving about in the water. Such a picture is obviously fictitious, since you could not have been looking at yourself, but images in the memory often include fleeting pictures of this kind. (p. 924)

Sabom expressed his skepticism explicitly, claiming that Moody "did not attempt to substantiate these reports (of visual details) by medical records or other available sources"' and proposing to do just that:

Now, the majority of patients whom I would be interviewing had been resuscitated from a cardiac arrest. By this time in my career, I had personally directed and participated in well over a hundred such resuscitations. I knew what a resuscitation consisted of, that is, how it would look. I was anxiously awaiting the moment when a patient would claim that he had "seen" what had transpired in his room during his own resuscitation. Upon such an encounter, I intended to probe meticulously for details that would not ordinarily be known to nonmedical personnel. In essence, I would pit my 
experience as a trained cardiologist against the professed visual recollections of lay individuals. In so doing, I was convinced that obvious inconsistencies would appear which would reduce these purported visual observations to no more than an "educated guess" on the part of the patient. (p. 7)

\section{Moreover,}

Twenty-five "control" patients were interviewed whose backgrounds were similar to those reporting autoscopic NDEs and who had been consecutively admitted to a coronary care unit (CCU) . . . While in the CCU, each of these patients had had the opportunity to observe closely at his bedside a cardiac monitor to which he was attached, a cardiac defibrillator, and intravenous needles and equipment. Moreover, each patient had admitted to regular viewing of a home television set prior to this admission. Thus this group of twenty-five cardiac patients had received considerable exposure to hospital routine and television programs, both of which could have contributed to their knowledge of CPR.

During the interview, each patient was asked to imagine that he was standing in the corner of a hospital room watching a medical team revive a person whose heart had stopped beating. He was then asked to describe in visual detail what he would expect to see in such a situation. He was cautioned to describe only those details that he was reasonably confident would actually be seen during CPR on a hospitalized patient. Each of these interviews was tape-recorded and later analyzed.

Twenty-three of the twenty-five interviewed patients made some attempt to describe the CPR procedure based on their own general knowledge of hospital equipment and protocol. Without undue prompting, twenty of these twenty-three respondents made a major error in their descriptive accounts. (pp. 84-85)

In contrast, the 32 surgery-related prospective NDE cases in Sabom's study provided autoscopic descriptions that "did correspond in at least a general way to the known facts of the near-death crisis event," and 6 of these 32 survivors "were able to record specific details of their near-death crisis event" (p. 87). These were the cases for which Sabom had been skeptically and "anxiously awaiting." The result: Their recollections were in fact verified in fine-grained detail from medical records and Sabom's own technical knowledge. Further, the recollected details in each case were "fairly specific for the actual resuscitation being described and . . . not interchangeable with the clinical circumstances of other near-death crisis events"' (p. 114). One NDE survivor did make apparent errors in his description of the working of a defibrillating meter-until Sabom discovered that the recollection described precisely the working of an older model that was still "in common use in 1973, at the time of his [the subject's] cardiac arrest"' (p. 104). Sabom considered several possible explanations for the accuracy of the recollections, including the possibility "that the accuracy of the NDE 
could be attributed to information passed on to the near-death survivor by someone (a doctor, a nurse, etc.) who witnessed the resuscitation":

I find this possibility unlikely for two reasons. First, the type of information contained in the autoscopic descriptions is not what would likely be explained to a patient recovering from a cardiac arrest. It is usually appropriate to explain to resuscitated patients that their "heart stopped beating" and that an "electrical shock" was used on the chest to stabilize cardiac rhythm, but there is no conceivable reason to supply the details reported in the typical autoscopic NDE-the insertion of a plastic airway, the checking for a carotid pulse or pupillary response in the eye, the drawing of arterial blood from the hand or the groin, the movement of the needles on the face of the defibrillator, etc. Second, several patients claimed that they reported the autoscopic experience soon after the resuscitation. Interviews with family members confirmed these claims. Moreover, these family members had observed that the original description of the NDE was consistent with subsequent retellings of the experience by their resuscitated relative. (p. $114)^{7}$

\section{STATUS AND EFFECTS UNIQUELY ATTRIBUTABLE TO NDEs}

According to Moody, NDErs report the NDE to be quite real (in contrast to dreams or hallucinations), to have inspired them to love others in a deeply empathic way, and to have convinced them that there is life after death. One survivor is quoted as saying: "It was real . . . My mind wasn't at that point where I wanted to make things happen or make up anything. I just wasn't in the state of mind" (1975, p. 84). Another stated: "It was nothing like a hallucination. I have had hallucinations once, when I was given codeine in the hospital. But that had happened long before the accident which really killed me. And this experience was nothing like the hallucinations, nothing like them at all' (pp. 84-85). Many respondents found their lives "broadened and deepened" (p. 89) by this quite real experience. As one survivor stated:

I try to do things that have more meaning and that make my mind and soul feel better. And I try not to be biased, and not to judge people. I want to do things because they are good, not because they are good to me. And it seems that the understanding I have to things now is so much better. I feel like this is because of what happened to me, because of the places I went and the things I saw in this experience. (p. 90)

Finally, according to Moody, "death is no longer frightening," because "after his experience a person no longer entertains any doubts about his survival of bodily death. It is no longer merely an 
abstract possibility to him, but a fact of his experience" (p. 96).

Siegel, while accepting Moody's descriptions of aftereffects, did not regard such aftereffects as unique to the NDE. Siegel noted that hallucinations can seem quite real and vivid, and can engender "a feeling of insight" as well as "changed attitudes and beliefs that pass into an afterglow and remain as a vivid memory" (p. 924). However, Siegel would presumably doubt that claim that experiencers of both hallucinations and an NDE would consistently distinguish the latter as uniquely real and impactful.

We can ask these questions: Was Moody correct that the NDE is typically recollected as real (distinguishable from dreams or hallucinations) and impactful? Is there replication that those NDE survivors who have also experienced hallucinations regard the experience as distinguishable? Are the supposed effects on subsequent life specifically attributable to the NDE, or are they inspired by any near-death crisis event, NDE or otherwise? Findings by Ring and Sabom speak to these issues.

The recent research does substantiate that respondents do perceive the NDE as reality. In the Ring sample, 19 out of 22 respondents asked whether the experience was like a dream stated emphatically that it was not ( 2 were unsure, and 1 stated that it was like a dream): e.g., "It was too real. Dreams are always fictitious. This was me, happening at that time and there was no doubt that it was reality' (1980, p. 82; cf. Gallup, 1982, pp. 39-41). Regarding the respondents' evaluation relative to hallucinations, Ring's result generally supported Moody:

I did find some evidence for the occurrence of hallucinatory-like images among a small number of our respondents-there were perhaps a half dozen such cases-including both core experiencers and nonexperiencers. In every case, however, the hallucinatory images were completely idiosyncratic and were regarded afterward by the respondents as having been hallucinations, that is, not real. In the few instances where a core experiencer also reported having had hallucinations, these could be distinguished from the core experience itself as having had a distinctly different quality. Perhaps the most definitive comment was delivered by one of our core experiencers, who was herself a psychiatrist and who, accordingly, should know something both about dreams and hallucinations. She told me, without qualification, that, in her judgment, her own experience was neither the one nor the other. (p. 83)

Sabom's results also corroborated Moody's assertions on this point. Indeed, one respondent regarded his NDE as "realer than here, really. After that the world seemed like a mockery to real life"' (p. 16). Another respondent reported: 
I was in a coma for seven or eight days and I had all those other convulsions. I had hallucinations then but they weren't the same. They were real-that is, they weren't like a dream, yet they weren't the same as I felt in that ambulance, in that in the hallucinations I'd be more like a spectator but in this experience [the NDE] where I lifted out of my body, it was me! (p. 169)

Moody also stated (and Siegel did not doubt) that the NDEs had had considerable impact on the survivors in many instances. This assertion is supported by the recent research, but only in certain respects when the effects are compared to those reported by nonNDE near-death survivors. Ring, using retrospective questionnaire data, reported that 37 percent of the NDE respondents attributed to the experience an increased appreciation of life; 24 percent, a "renewed sense of purpose"; and 24 percent felt "more loving, caring." 8 The corresponding percentages for the nonexperiencers were 29,20 , and 12 , but these differences favoring the NDE survivors were not significant. Nor were other suggestive percentage differences: "stronger person," 20 percent vs. 8 percent; "more patient, understanding," 10 percent vs. 0; "want to help others," 8 percent vs. 0 . Ring did note his "personal" and qualitative", impression, however, that

experiencers are more likely to show a heightened sense of what I can only vaguely call "spiritual awareness" and this quality seems to pervade the other changes that they report. They also seem, more often, to radiate a certain serenity or peace or acceptance of life. (1980, p. 140)

Statistically significant differences were found in reorientation of views on religion and death. NDE survivors recollected significantly more change than non-NDE survivors on "religious feeling" ( $p$. 159), as well as on a "global religiousness" scale (attributable mainly to a "belief in life after death component, but enhanced by a "belief in God" component). Also, there was a dramatic retrospective decline in "fear of death" for experiencers relative to nonexperiencers. Finally, asked to say "what death meant to them," 37 percent of the experiencers (vs. 5 percent of the nonexperiencers) mentioned "peace, beauty, or bliss," and 24 percent of the experiencers (vs. 4 percent of the nonexperiencers) mentioned reincarnation as at least a possibility.

Sabom's results were similar. He reported, for the experiencers relative to nonexperiencers in his sample, highly significant declines in fear of death (in terms of both questionnaire and interview data) and highly significant increases in belief in afterlife (pp. 212-213). Also, Sabom noted: 
In my medical involvement in the lives of many of these patients, I could often detect a change in the attitudes and beliefs of those who had told me of their NDE. A deepening of religious beliefs, a changing of vocational interest (e.g., becoming a hospital volunteer) and a focus on more humanitarian concerns were common developments. Moreover, a patient's outlook on life was often transformed in such a way as to allow him to cope more successfully with the day-to-day trials of a medical illness . . . . Each person would invariably attribute the change in some way to the NDE. (p. 157; cf. Gallup, 1982, pp. 128-134)

In summary, the NDE is evidently experienced as reality and does generate certain changes in worldview and lifestyle, even when compared to effects attributable to a close brush with death (some differences are not significant, however). Such a confirmation merely supports Siegel's acceptance of Moody's claims to this effect, however, and may not be discriminative of an imaginal (hallucinatory) vs. veridical (spiritually authentic) interpretation. On the other hand, somewhat discouraging of the hallucination hypothesis are the cases cited by both Moody and the subsequent researchers wherein experiencers of both hallucinations and NDEs emphatically distinguished the latter and accorded it reality status.

\section{CONCLUSION}

Overall, the recent research tends to support Moody's original inferences and even his conclusion from this phenomenon that "death is a separation of the mind from the body, and that mind does pass into other realms at this point'"(1975, p. 151). Moody is supported that the NDE is fairly common and coherent, that it is in fact a relatively robust phenomenon cutting across various background and circumstantial factors, that its likelihood ${ }^{9}$ and extensiveness are associated with degree of physical near-death, that reported visual and auditory recollections can be independently corroborated, and that it is for the survivor a quite real and uniquely impactful experience (at least in certain respects). Ring's and Sabom's own conclusions concur with Moody's. Ring: "Consciousness (with or without a second body) may function independently of the physical body" (1980, p. 233). Sabom pondered an affirmative answer to the question: "Is out-of-body perception indeed occurring during the NDE, and if so, is some element of the human organism (the mind?) separating from the physical determinants of consciousness (the brain?) to accomplish such a feat?'" (p. 181).

Certainly, Siegel's hallucination hypothesis encounters some turbulence in light of recent research. Sabom, too, had "suspected that the experience would largely reflect personal dreams and 
fantasies"' but was forced to reconsider upon finding "basic patterns" (p. 158) as well as "verification" (p. 159) of the autoscopic observations. Further, the recent research consistently supports Moody's assertion that survivors who have also experienced hallucinations emphatically claim the distinguishability of the NDE. Finally, Siegel's process hypothesis of neural excitation and arousal runs into particular difficulty against findings that the likelihood and extensiveness of the NDE are associated with the pronounced neurophysiologically depressive characteristics of clinical death.

A hypothesis that an imaginal elaboration in some sense is involved in the NDE may not be totally discountable, however. Sabom acknowledges that the NDE "can be entered into and modified in certain individual ways"' (p. 16). Indeed, in at least one instance, the "presences"' encountered by an NDEr were quite this-worldly and possibly delusional products ("four nurses" engaging in a "verbal interrogation" about the experiencer's "possible "subversive activities"'). Also, Ring acknowledged "that what is seen is, at least at first, largely determined by preexisting schemata of near-death survivors" (1980, p. 248).

It is also clear, however, that a subjectivist interpretation cannot provide a total explanation. I state this chiefly because of the rather astonishing controlled evidence found by Sabom for the verifiability of the NDE autoscopic component. The experiential validity of the autoscopic component then bids that we take the transcendental component seriously. As Sabom pointed out: "In the combined NDE, the transcendent portion occurs in sequence following the autoscopic elements and is perceived by the person as being equally vivid and real as its autoscopic forerunner' (p. 185). Sabom's speculation: "Since I suspect that the NDE is a reflection of a mindbrain split, I cannot help but wonder why such an event should occur at the point of near-death. Could the mind which splits apart from the physical brain be, in essence, the 'soul,' which continues to exist after final bodily death, according to some religious doctrines?' (p. 185). That is where we, at this point in the research, must leave the matter: there is indeed evidence of spiritually suggestive life at the point of bodily death.

\section{NOTES}

1. Siegel did not rule out the possibility, however, that the "objective" interpretation could be correct: "Finding parallels with satisfactory explanations of [one's] own is not the same as finding proof against [spiritual] survival" (p. 927). 
2. As Ring (personal communication, June 2, 1984) has pointed out, Sabom's use of the term "autoscopic" should not be confused with the standard meaning of autoscopic projection, referring to the "observation" of one's body image from the standpoint of one's physical body.

3. In a later study of 32 suicide survivors, Ring and Stephen Franklin (1982) noted a minor but interesting qualitative variation that "has occurred, to the best of our knowledge, only three times in all our NDE cases but each time in a suicide survivor," namely, "drifting through a grayness rather than a blackness or darkness." In Life at Death (1980), Ring also quoted one suicide survivor's recollection "describing a portion of a conversation (in thought) he had, while unconscious, with what he took to be God.

... then He said, "Do you want to go back?" And He goes, "Finish your life on earth." And I go, "No, I want to die." And He goes, "You are breaking my laws to commit suicide. You'll not be with me in heaven if you die." And I say, "What will happen?" And then after this I started coming to. So I don't know what happened after this. So I think that God was trying to tell me that if I commit suicide I'm going to go to hell, you know? So, I'm not going to think about suicide anymore (laughs nervously). (p. 127)

Similarly, according to Moody (1975), his "few cases" of suicide survival stated that "in their disembodied state they were unable to do anything about their problems, and they also had to view the unfortunate consequences which resulted from their acts"' (p. 143).

4. Unfortunately, there is at present no cross-cultural research on the NDE (although there are some suggestive similar results from a comparative [Indian and American] study of death-bed visions; Osis and Haraldsson, 1977). Moody noted some quite remarkable parallels to the NDE in the ancient Tibetan Book of the Dead, as well as in the near-death recollections of a seventeenth-century Swedish scientist-turned-visionary, Emanuel Swedenborg.

5. Maurice Rawlings (1978), a cardiologist, has reported learning among his dying and resuscitated patients of numerous "bad" (hellish or nightmarish) "NDEs" (cf. Gallup, 1982, pp. 73-87). Rawlings also noted, however, that the content of the hellish cases "seems to vary considerably . . . from case to case," unlike the similarity across his "good" cases (p. 90). This observation raises a question as to whether the "hellish" reports 
should be considered to be intrinsic to the NDE phenomenon (cf. Ring, 1980, pp. 193-195, 248-250).

6. This statement is accurate only if one assumes misrepresentation by Moody, who claimed that he did investigate medical records in some cases, and "the records have borne out the assertions of the persons involved ... that when medical records have not been usable, I have secured the testimony of others-friends, doctors, or relatives of the informant-to the effect that the neardeath event did occur'" (p. 146).

7. Other naturalistic explanations considered and rejected by Sabom (1982) included: semiconscious auditory perception, conscious or unconscious fabrication, depersonalization, autoscopic hallucination, dreams, fulfillment of expectation, drug-induced delusion, endorphin release, temporal lobe seizure, hypoxia, and hypercarbia (pp. 151-178).

8. In a more recent study restricted to aftereffects in survival of deep NDEs, Ring (1984) concluded that these persons are especially "likely to show greater appreciation for life and more concern and love for their fellow humans while their interest in personal status and material possessions wanes" (p. 141).

9. The determinants of individual differences in the likelihood of having an NDE upon near death are largely a matter of speculation at this point. It is known that the NDE's likelihood and extensiveness are associated with a relatively serious neardeath episode (see text). Yet among those who have come close to death on numerous occasions, some have had NDEs consistently, others only once or twice, still others not at all (Sabom, 1982). Perhaps experiences for these latter persons come only at the most "serious" point in the continuum, actual bodily death.

\section{REFERENCES}

Gallup, G., Jr. Adventures in Immortality: A Look Beyond the Threshold of Death. New York: McGraw-Hill, 1982.

Gibbs, J. C. The near-death experience: balancing Siegel's view. American Psychologist, 1981, 36, 1457-1458.

Moody, R. A. Life After Life. Covington, GA: Mockingbird, 1975. Moody, R. A. Reflections on Life After Life. San Francisco: Cameron and Co., 1977.

Osis, K., and Haraldsson, E. At the Hour of Death. New York: Avon Books, 1977. 
Rawlings, M. Beyond Death's Door. Nashville, TN: Thomas Nelson, 1978.

Ring, K. Life at Death: A Scientific Investigation of the Near-Death Experience. New York: Coward, McCann, \& Geoghegan, 1980.

Ring, K. Heading Toward Omega: In Search of the Meaning of the Near-Death Experience. New York: Morrow, 1984.

Ring, K., and Franklin, S. Do suicide survivors report near-death experiences? In C. R. Lundahl (Ed.), A Collection of Near-Death Research Readings. Chicago: Nelson-Hall, 1982.

Sabom, M. B. Recollections of Death: A Medical Investigation. New York: Harper \& Row, 1982.

Siegel, R. K. The psychology of life after death. American Psychologist, 1980,35, 911-931.

Woodhouse, M. B. Five arguments regarding the objectivity of NDEs. Anabiosis, 1983, 3, 63-76.

Requests for reprints to:

John C. Gibbs

Department of Psychology

Ohio State University

142 Townshend Hall

1885 Neil Ave.

Columbus, OH 43210 